\title{
STABILITY CHARACTERIZATION OF THE RESPONSE OF WHITE STORKS' FORAGING BEHAVIOR TO VEGETATION DYNAMICS RETRIEVED FROM LANDSAT TIME SERIES
}

\author{
Ines Standfuß $\beta^{1}$, Christian Gei $\beta^{1}$, Ran Nathan ${ }^{2}$, Shay Rotics ${ }^{2}$, Stefan Dech ${ }^{1}$ and Hannes Taubenböck ${ }^{1}$ \\ ${ }^{1}$ German Remote Sensing Data Center (DFD), German Aerospace Center (DLR), Wessling, Germany \\ ${ }^{2}$ Movement Ecology Laboratory, Department of Ecology, Evolution and Behaviour, Alexander \\ Silberman Institute of Life Sciences, The Hebrew University of Jerusalem, Jerusalem, Israel
}

\begin{abstract}
Agricultural activities cause rapid changes in vegetation development at local and regional scales. Those modifications affect the small-scale behavior of animals, like the foraging ground usage of breeding white storks. Only recently, a novel approach, that enables to quantify the relationship between mowing and harvesting activities and a prolonged foraging time of storks by combining remote sensing time series with GPS telemetry, has been proposed. This study examines the stability of this approach. We investigate two potential influencing factors: different vegetation indices and time lags over which vegetation dynamics were retrieved. Mostly independent from the vegetation index and time lag, we observed that storks spent large proportions of foraging time in areas characterized by a recent drop in vegetation indices, indicative for a preferred usage after harvesting and mowing events. This suggest that the proposed approach is relatively stable and hence, provides a reasonable basis to investigate the effects of anthropogenic vegetation alterations on animal behavior at small spatiotemporal scales.
\end{abstract}

Index Terms - remote sensing, telemetry data, vegetation dynamics, Anthropocene, habitat usage

\section{INTRODUCTION}

Man-made alterations of the environment, e.g. ongoing land transformations and climate change, pose a major threat to global wildlife [1]. A comprehensive and detailed understanding of the impact of such processes on various aspects of animal life and, in the long term, on survival of species is still in its infancy. Combining remote sensing and telemetry data enables to link environmental characteristics to movement trajectories, behavior and habitat use of animals [2]. This provides an unprecedented opportunity to thoroughly assess how wildlife copes with anthropogenic environmental alterations.

A well-researched topic often investigated using a combination of remote sensing time series and telemetry data, is the "green wave hypothesis". Studies focusing on this subject aim to identify the influence of vegetation green-up on the choice of foraging areas made by herbivores during migration [3], [4]. These investigations have one element in common: they mainly consider the influence of seasonal vegetation growth cycles on animal behavior.

However, vegetation development at regional and local scales is, beyond seasonal patterns, strongly affected by human activities such as harvesting cycles. These practices can significantly impact animal behavior though mainly at small spatial scales. In-situ observations of breeding white storks, for instance, revealed prolonged foraging on cropland and grassland fields after management practices such as harvesting or mowing [5]. However, a method to properly quantify this animal-environment relationship by other means than observations did not exist until recently.

The currently active Landsat 7 and 8 satellites repeatedly record the Earths' surface every 8 days with a spatial resolution of $30 \mathrm{~m}$ in the optical domain. Those spatial and temporal properties are suitable to retrieve intraannual profiles of vegetation indices, like the NDVI, at field scales and enable to identify abrupt changes, like a drop in vegetation indices as induced by harvesting and mowing practices [6], [7]. State of the art telemetry collars like those provided by the e-obs $\mathrm{GmbH}$ allow for tracking animal movement with 5-minute resolution [8] and hence, facilitate to retrieve detailed information on daily foraging ground usage of white storks. With regard to this, [9] proposed a novel approach, relying on a combination of Landsat time series and e-obs telemetry data, to systematically capture small-scale, man-made vegetation changes and to investigate their effects on white storks' foraging behavior. In line with the in-situ observations, they found that white storks spent large amounts of foraging time at fields characterized by a recent drop in NDVI, indicative for a frequent usage after mowing and harvesting.

In this study, we aim to investigate the stability of this approach. We focus on two influencing factors, differing vegetation indices and time lags over which vegetation dynamics are measured. We want to test if the observed foraging ground preferences of white storks can be reproduced with different data combinations. 


\section{STUDY AREA}

The study area comprises the breeding areas of the 18 considered white storks. Those are located at the northeastern part of Germany in the vicinity to Loburg $\left(52^{\circ} 7^{\prime} \mathrm{N}\right.$ $\left.12^{\circ} 4^{\prime} \mathrm{E}\right)$, Beuster $\left(52^{\circ} 55^{\prime} \mathrm{N} 11^{\circ} 46^{\prime} \mathrm{E}\right)$, and the Drömling Nature Park $\left(52^{\circ} 28^{\prime} \mathrm{N} 11^{\circ} 7^{\prime} \mathrm{E}\right)$. This region is characterized by high shares of agricultural areas and moderate amounts of grassland.

\section{METHOD}

\subsection{Derivation of vegetation indices from remote sensing time series on field scale}

To characterize the intra-annual vegetation development, we used a time series of Landsat 7 and Landsat 8 Top-ofAtmosphere data with cloud coverage $<100 \%$ for the year 2014. Initially, we applied the ATCOR-2 software [10], to correct atmospheric effects and to derive surface reflectance. Subsequently, we identified pixels which were unusable due to opaque clouds, haze, radiometric saturation or, in case of Landsat 7, affected by the Scan-Line Corrector failure [11] with the Landsat Quality Assessment band. To accommodate for differences in the spectral resolution between the Landsat 7 and Landsat 8 spectral bands, we normalized the Landsat 7 surface reflectance to the Landsat 8 surface reflectance. This was accomplished by applying the approach proposed by [12].

After completion of these steps, we calculated four vegetation indices (VI) for every image of the Landsat time series. These comprised the NDVI [13], EVI [14], SAVI [15] and MSAVI2 [16]. We utilized a data model containing vectorized landscape objects [17] as basis for field boundaries. Subsequently, we spatially linked these boundaries to a landcover classification [18] to identify agricultural and grassland fields. We combined the fields with the previously generated VI time series and calculated the spatial median VI for each field and time step using the pixels located within a field boundary. In a final step, we estimated a time series of daily VI values per field using Thin-Plate Spline smoothing [19].

\subsection{Derivation of foraging ground usage from telemetry data on field scale}

We used e-obs telemetry data of 18 breeding white storks to characterize their foraging ground usage. This data was collected within a preceding research project and included individual locations (5-min temporal resolution) with classified movement behavior from 2014 [8]. For every bird, we excluded the spring and autumn migration periods and retained solely locations which were classified as "walking" or "resting". The latter two mirror the foraging behavior of white storks which is characterized by walking movements and short resting periods [20]. Subsequently, we assigned the foraging locations to individual foraging bouts. To generate more robust representations of the habitat usage from the location data, we fitted a movement model to every foraging bout following the approach suggested by [21]. We used these models to simulate 1000 almost continuous movement paths with a 15 -second temporal resolution per foraging bout. In a final step, we calculated the (median) daily time spent within a field, by a spatial overlay of the individual simulation sets with the boundaries of the cropland and grassland fields.

\subsection{Stability characterization of the effect of vegetation dynamics on foraging behavior}

Agricultural management activities like harvesting and mowing cause VI to decrease [6], [7]. To make this abrupt change measurable, we calculated the amplitude of VI change for a time period of one up to 50 days before a registered foraging visit on a field as proposed by [9]. The amplitude is defined as the difference between the maximum and minimum VI. It takes a negative form if the maximum occurred earlier in time than the minimum. Fields visited after harvesting or mowing should be characterized by low VI and a negative amplitude of VI change.

The characteristics of a field were defined by the VI registered during a visit and the amplitude of VI change registered (one up to 50 days) before a visit. We used the amplitude value 0 to discriminate between negative and positive amplitude of VI change. To separate between low and high VI, we computed the mean between the $10^{\text {th }}$ and $90^{\text {th }}$ percentile of all VI values registered on the fields during foraging visits. We did this separately for grassland and cropland as well as the four considered VI. Next, we computed the percentual proportions of the total time the white storks spent foraging in fields with four different characteristics defined by the VI (low/high) and amplitude of VI change (negative/positive). We did this for all considered VI and time lag combinations. Finally, we compared the relative proportional shares of foraging time among those different data combinations.

\section{RESULTS}

On cropland, we observed the highest proportion of white storks foraging time at fields with low VI and negative amplitude of VI change (Fig. 1 - left column). This result was independent of 1) the considered VI and 2) the time lag for which the amplitude of VI change was computed.

On grassland, we observed large proportions of foraging time in fields with low VI and negative VI change as well as in fields with high VI and positive VI change (Fig. 1 - right column). This result was inconclusive when the amplitude of VI change was calculated for periods of less than $\sim 10$ days before a visit. However, it became stable with larger time lags and was independent from the utilized VI.

Both, the findings for cropland and grassland confirm the results highlighted by [6]. The proposed approach to 


\section{CROPLAND}
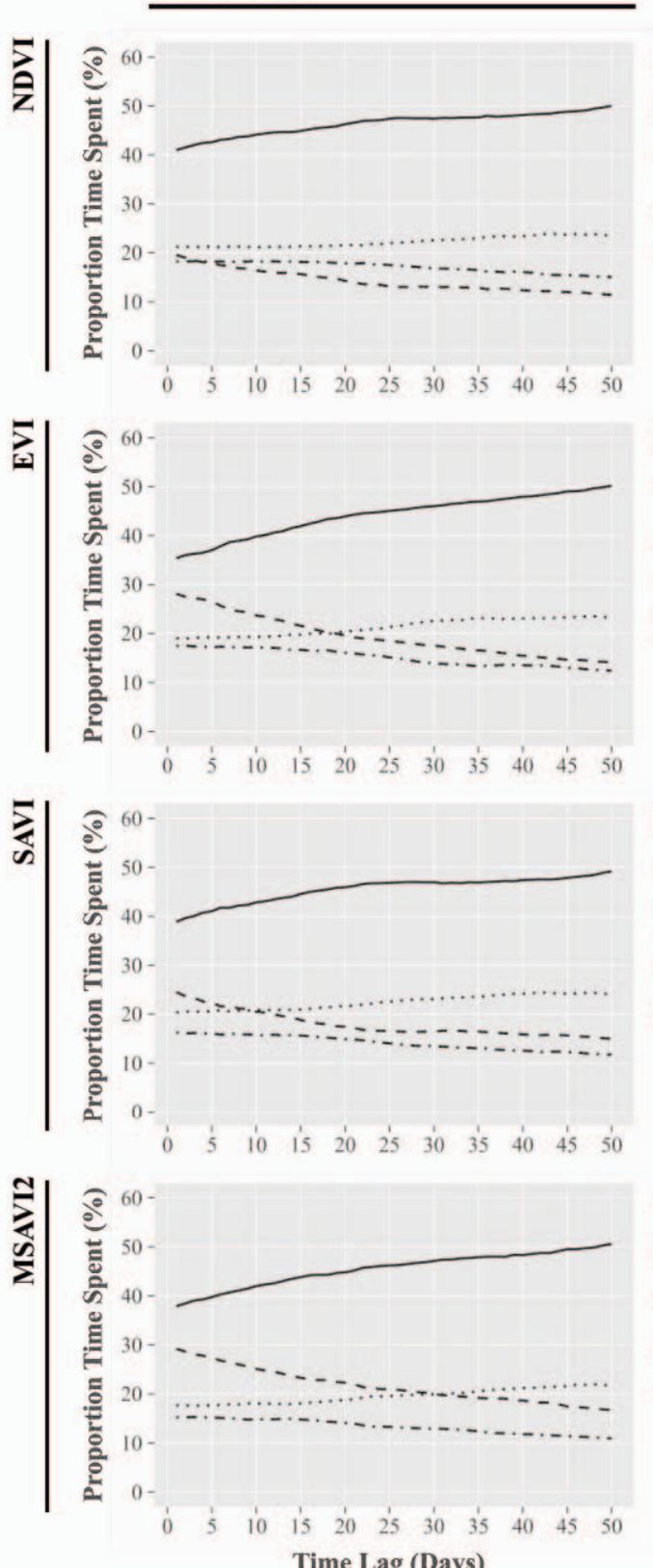

Legend

- - - low VI, positive amplitude of VI change

\section{GRASSLAND}
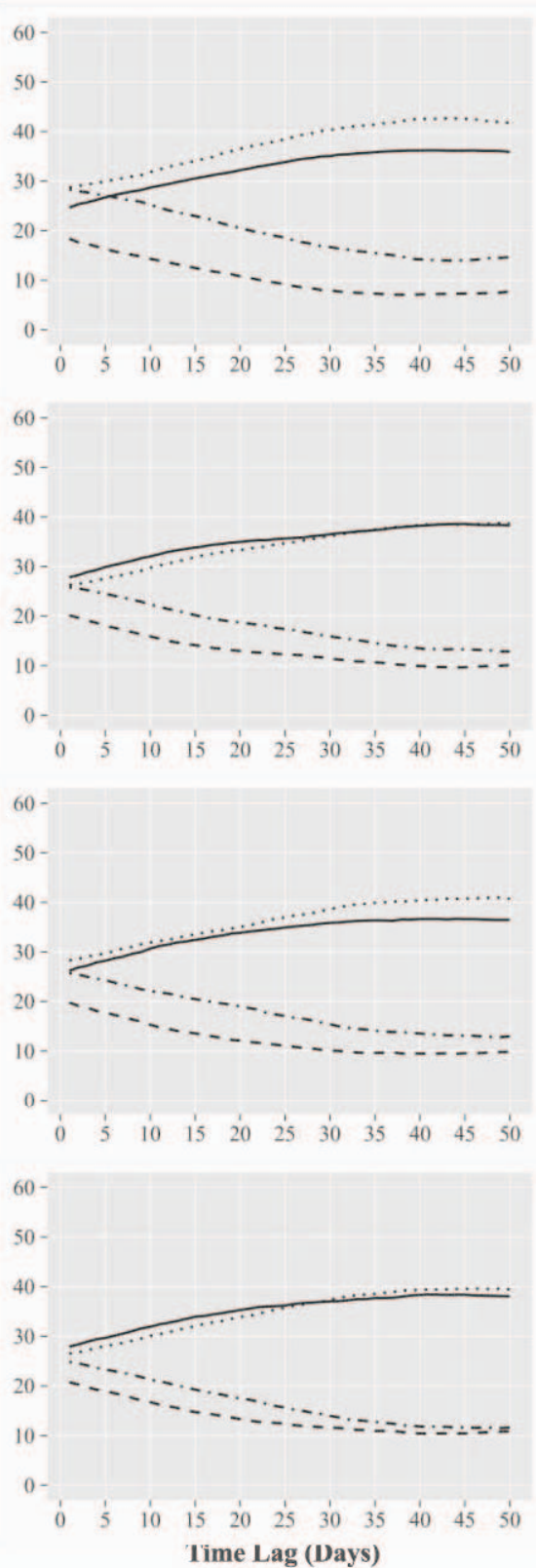

... high VI, positive amplitude of VI change

Fig. 1: Percentual proportions of the total time white storks spent foraging in fields with four different characteristics defined by the VI (low/high) and amplitude of VI change (negative/positive) registered during and before a visit, respectively. The xaxis displays the time lags from one up to 50 days before a visit for which the amplitude of VI change was calculated. Each row represents the results of the quantification for a different VI. The columns separate between cropland (left-hand side) and grassland fields (right-hand side).

uncover the influence of anthropogenic vegetation dynamics on foraging behavior of white storks provides thus, no matter which data combinations are utilized, relatively stable results.

\section{CONCLUSION}

Our findings emphasize that high spatiotemporal resolution vegetation index time series provide reliable means to investigate the influence of small-scale 
anthropogenic environmental changes on foraging behavior of white storks. The approach proposed by [9] should, beyond the white stork, be similarly suited to investigate how human-activities affect behavior and habitat usage of other species. In light of agricultural intensification and the increasingly threatened farmland species, such an approach could aid to build a knowledge basis for conservation management.

\section{REFERENCES}

[1] IPBES, Summary for policymakers of the global assessment report on biodiversity and ecosystem services of the Intergovernmental Science-Policy Platform on Biodiversity and Ecosystem Services. Bonn, Germany, 2019.

[2] R. Nathan et al., "A movement ecology paradigm for unifying organismal movement research," PNAS, vol. 105, no. 49, pp. 19052-19059, 2008.

[3] X. Wang et al., "Stochastic simulations reveal few green wave surfing populations among spring migrating herbivorous waterfowl," Nat. Commun., vol. 10, no. 1, 2019.

[4] J. A. Merkle et al., "Large herbivores surf waves of green-up during spring," Proc. R. Soc. B Biol. Sci., vol. 283, no. 1833, pp. 1-8, 2016.

[5] K. Johst, R. Brandl, and R. Pfeifer, "Foraging in a Patchy and Dynamic Landscape : Human Land Use and the White Stork," Ecol. Appl., vol. 11, no. 1, pp. 60-69, 2001.

[6] S. Estel, T. Kuemmerle, C. Levers, M. Baumann, and P. Hostert, "Mapping cropland-use intensity across Europe using MODIS NDVI time series," Environ. Res. Lett., vol. 11, no. 2, 2016.

[7] P. Griffiths, C. Nendel, J. Pickert, and P. Hostert, "Towards national-scale characterization of grassland use intensity from integrated Sentinel-2 and Landsat time series," Remote Sens. Environ., no. March 2018, pp. 1-12, 2019.

[8] S. Rotics et al., "The challenges of the first migration: movement and behaviour of juvenile vs. adult white storks with insights regarding juvenile mortality," J. Anim. Ecol., vol. 85, pp. 938-947, 2016.

[9] I. Standfuß, C. Geiß, R. Nathan, S. Rotics, and H. Taubenböck, "High-resolution time series analysis reveals effects of agricultural activities on habitat preferences (submitted)."

[10] R. Richter and D. Schläpfer, "Atmospheric / Topographic Correction for Satellite Imagery (ATCOR - 2/3 User Guide)," 2016.

[11] F. Chen et al., "The integrated WRF/urban modelling system: Development, evaluation, and applications to urban environmental problems," Int. J. Climatol., vol. 31, no. 2, pp. 273-288, 2011.

[12] D. P. Roy et al., "Characterization of Landsat-7 to
Landsat- 8 reflective wavelength and normalized difference vegetation index continuity," Remote Sens. Environ., vol. 185, pp. 57-70, 2016.

[13] J. W. Rouse, R. H. Hass, J. A. Schell, and D. W. Deering, "Monitoring vegetation systems in the great plains with ERTS," in Third Earth Resources Technology Satellite (ERTS) symposium, 1973, pp. 309-317.

[14] A. Huete, K. Didan, H. Miura, E. P. Rodriguez, X. Gao, and L. F. Ferreira, "Overview of the radiometric and biopyhsical performance of the MODIS vegetation indices," Remote Sens. Environ., vol. 83, pp. 195-213, 2002.

[15] A. R. Huete, "A soil-adjusted vegetation index (SAVI)," Remote Sens. Environ., vol. 25, pp. 295309, 1988.

[16] J. Qi, A. Chehbouni, A. R. Huete, Y. H. Kerr, and S. Sorooshian, "A modified soil adjusted vegetation index," Remote Sens. Environ., vol. 48, pp. 119126, 1994.

[17] BKG, Ed., "Digitales Landbedeckungsmodell für Deutschland - LBM-DE2012," 2016, pp. 1-18.

[18] B. Mack, P. Leinenkugel, C. Kuenzer, and S. Dech, "A semi-automated approach for the generation of a new land use and land cover product for Germany based on Landsat time-series and Lucas in-situ data," Remote Sens. Lett., vol. 8, no. 3, pp. 244-253, 2017.

[19] J. Duchon, "Splines minimizing rotation-invariant semi-norms in sobolev spaces," in Constructive Theory of Functions of Several Variables, W. Schempp and K. Zeller, Eds. Berlin Heidelberg: Springer, 1977, pp. 85-100.

[20] L. M. Carrascal, J. C. Alonso, and J. A. Alonso, "Aggregation size and foraging behaviour of white storks Ciconia ciconia during the breeding season," Ardea, vol. 78, no. 3, pp. 399-404, 1990.

[21] C. H. Fleming, J. M. Calabrese, T. Mueller, K. A. Olson, P. Leimgruber, and W. F. Fagan, "From Fine-Scale Foraging to Home Ranges: A Semivariance Approach to Identifying Movement Modes across Spatiotemporal Scales," Am. Nat., vol. 183, no. 5, pp. E154-E167, 2014. 